\title{
Efectos de los laboratorios de ciencias con tic en la comprensión y representación de los conocimientos científicos en estudiantes del bachillerato en un contexto escolar cotidiano
}

\author{
Fernando Flores-Camacho, Leticia Gallegos-Cázares, Beatriz-Eugenia García-Rivera y \\ Araceli Báez-Islas
}

\section{RESUMEN}

Este trabajo da respuesta a la interrogante de si los recursos tecnológicos tienen alguna influencia en la comprensión que alcanzan los alumnos cuando las condiciones en las que se aplican no tienen control experimental, sino que simplemente son utilizados en función de la visión y capacidad tecnológica de cada profesor. Los datos y análisis indican que la introducción en las escuelas de laboratorios de ciencias con una gran variedad de recursos tecnológicos que favorecen un entorno multi-representacional, tiene influencia positiva en la comprensión y posibilidad de representación que logran los alumnos en biología y física, en situaciones diversas de aproximación didáctica de los profesores.

Palabras clave: enseñanza de la ciencia, bachillerato, tecnologías de la información y la comunicación, laboratorios de ciencias, representaciones científicas, México.

\section{Fernando Flores-Camacho}

fernando.flores@ccadet.unam.mx Mexicano. Doctor en Pedagogía, Universidad Nacional Autónoma de México (UNAM). Académico del Grupo de Cognición y Didáctica de las Ciencias, Instituto de Ciencias Aplicadas y Tecnología, UNAM, Profesor del Posgrado de Pedagogía, UNAM. Temas de investigación: transformación y construcción conceptual, didáctica de las ciencias.

\section{Leticia Gallegos-Cázares}

leticia.gallegos@ccadet.unam.mx Mexicana. Doctora en Pedagogía, Universidad Nacional Autónoma de México (UNAM). Académica del Grupo de Cognición y Didáctica de las Ciencias, Instituto de Ciencias Aplicadas y Tecnología, UNAM, Profesora del Posgrado de Pedagogía, UNAM. Temas de investigación: transformación y construcción conceptual, didáctica de las ciencias, enseñanza de las ciencias en comunidades indígenas.

Beatriz-Eugenia García-Rivera

beatriz.garcia@ccadet.unam.mx Mexicana. Doctora en Pedagogía, Universidad Nacional Autónoma de México (UNAM). Académica del Grupo de Cognición y Didáctica de las Ciencias, Instituto de Ciencias Aplicadas y Tecnología, UNAM. Temas de investigación: enseñanza de la biología, transformación y construcción representacional, multirrepresentacionalidad, multiculturalidad.

\section{Araceli Báez-Islas}

baezi.araceli@gmail.com

Mexicana. Maestría en Docencia para la Educación Media Superior-Biología, Universidad Nacional Autónoma de México (UNAM). Profesora, Escuela Nacional Preparatoria 9 "Pedro de Alba”, unAM. Temas de investigación: enseñanza de la biología, transformación y construcción representacional, multirrepresentacionalidad, multiculturalidad. 
Efeitos dos laboratórios de ciências com TIC na compreensão e representação dos conhecimentos científicos em estudantes do bacharelado em um contexto escolar cotidiano

\section{RESUMO}

influência na compreensão que atingem os alunos quando as condições nas que se aplicam não têm controle experimental, mas simplesmente são utilizados em função da visão e capacidade tecnológica de cada professor. Os dados e a análise indicam que a introdução nas escolas de laboratórios de ciências com uma grande variedade de recursos tecnológicos que favorecem um entorno multi-representacional, tem influência positiva na compreensão e possibilidade de representação que conseguem os alunos em biologia e física, em situações diversas de aproximação didática dos professores.

Palavras chave: ensino da ciência, bacharelado, tecnologias da informação e da comunicação, laboratórios de ciências, representações científicas, México.

\section{The Efects of Іст Science Laboratories on the Understanding and Representation of Scientific Knowledge in High School Students in an Everyday School Context}

\section{ABSTRACT}

This paper answers the question of whether technological resources have any influence on students' understanding or not when the conditions in which they are applied have no experimental control, but are simply used according to the vision and technological capacity of each teacher. Data and analysis indicate that the introduction in the school context of science laboratories with a great variety of technological resources that favor a multi-representational environment has a positive influence on the understanding and possibility of representation that students achieve in Biology and Physics, in diverse situations of didactic approach proposed by the teachers.

Key words: science teaching, high school, information and communication technologies, science laboratories, scientific representations, Mexico. 


\section{Introducción}

En las últimas décadas, la introducción de las tecnologías de la información y la comunicación (TIC) en las aulas ha sido motivo de numerosas investigaciones sobre su influencia en el aprendizaje y acerca de si los entornos tecnológicos constituyen un nuevo marco o escenario que implique cambios en los procesos educativos (Chandra y Lloyd, 2008; Rogers y Twidle, 2013; de Winter, Winterbottom y Wilson, 2010; Díaz-Barriga, 2013).

La enseñanza de las ciencias no es la excepción y en diversas investigaciones se ha analizado, de manera puntual, cómo diversas tecnologías digitales contribuyen al aprendizaje de los conocimientos científicos. Por ejemplo, hay estudios en los que se analiza el uso de simuladores (Geelan y Fan, 2013; Al-Balushi, Al-Musawi, Ambusaidi y Al-Hajri, 2017; Gale et al., 2016), otros se enfocan en el uso de sensores y las mediciones que con ellos se obtienen (Magana y Balachandran, 2017; Haglund, Jeppsson y Schönborn, 2016; Soderberg y Price, 2003; Tortosa, 2013). También se ha analizado la influencia de bases de datos y la elaboración de mapas conceptuales, por citar algunos otros recursos relevantes (Savinainen, Mäkynen, Nieminen y Viiri, 2017; Eliam y Gilbert, 2014; Rau, Aleven y Rummel, 2015).

En la mayoría de esos estudios, se presenta una propuesta didáctica específica para llevar al aula, la cual se compara con grupos control que no emplean los diseños didácticos que se usan con los grupos experimentales. Como es común en ese tipo de trabajos, los grupos que han tenido alguna forma de intervención generalmente muestran resultados positivos.

En esas investigaciones también es común que las propuestas de intervención con TIC cuenten con profesores o investigadores que tienen un amplio o aceptable dominio de las tecnologías con las que experimentan en el aula, mientras que los profesores de los grupos control no necesariamente tienen ese mismo dominio o deliberadamente no incorporan las tecnologías.
Estos aspectos, sin que demeriten que nuevos enfoques y propuestas son relevantes y que el uso de las TIC en el aula o el laboratorio son necesarios, no reflejan lo que ocurre en una gran mayoría de escuelas que, habiendo invertido en recursos digitales para sus aulas y laboratorios, no cuentan con profesores con amplio dominio tecnológico, o bien no tienen estrategias didácticas que sean seguidas al pie de la letra por todos los profesores, sino que por el contrario, cada profesor utiliza esos recursos de diversas maneras, con distintos enfoques educativos y con variada intencionalidad, en términos del aprendizaje de sus alumnos.

Los aspectos descritos, llevan a plantearse la interrogante de si los recursos tecnológicos tienen alguna influencia en la comprensión que alcanzan los alumnos cuando las condiciones en las que se aplican no tienen control experimental, sino que, simplemente, son utilizados en función de la visión y capacidad de cada profesor, es decir, cuando son utilizados por profesores con niveles de distinto dominio de los recursos tecnológicos (Calderón et al., 2016) y cuando se implementan en situaciones escolares cotidianas en las que hay gran diversidad de aproximaciones didácticas sobre el uso de las TIC en las aulas y laboratorios.

En el presente trabajo se analiza la posible influencia que tienen las tecnologías digitales de los laboratorios de ciencias en condiciones cotidianas, sin ningún tipo de intervención experimental, en la comprensión y posibilidades de representación que logran los estudiantes del bachillerato sobre los temas de Genética y Fuerzas y Movimiento. El análisis se hace desde un marco representacional aplicado a situaciones de aula.

\section{El marco de análisis representacional}

Una de las ventajas que ofrece el uso de las tecnologías digitales en la enseñanza de las ciencias es que facilitan la utilización de diversas formas de representación externa que contribuyen, de manera 
relevante, a la comprensión del conocimiento científico (Ainsworth, 2006; Cook, 2006; Schnotz y Bannert, 2003; Waldrip y Prain, 2013). A pesar de ello, la mayoría de los estudios sobre la influencia de las tecnologías digitales en la escuela ha centrado su atención en aspectos como la identificación y/o declaración de conceptos o su influencia en la motivación hacia las ciencias (Schnotz y Bannert, 2003; Pende y Chandrasekharan, 2017) y se ha analizado menos cómo los estudiantes construyen y transforman sus representaciones.

Las representaciones, sean en forma implícita (concepciones de procesos en la mente de los sujetos) o explícita (expresiones en cualquier forma: oral, gráfica y simbólica), son construcciones que se convierten en elementos para la interpretación de los conceptos, la construcción de explicaciones causales y analógicas, y en elementos para la intervención experimental en los fenómenos. Por ejemplo, Prain y Tytler (2013) han analizado desde una perspectiva socio-cultural las representaciones y su relación con los procesos de aprendizaje de los alumnos y han mostrado su influencia en los procesos colectivos de aprendizaje. También hay estudios históricos sobre el papel que han jugado las representaciones externas en el desarrollo de conceptos y teorías, como en el caso de Faraday, analizado por Nersessian (1990), que muestra cómo las representaciones previas sobre sus conceptos de materia y vacío influyeron en sus ideas de fuerza y campo eléctrico, o como el trabajo de Martí y Garcia-Milá (2007), que ha evidenciado que los esquemas que elaboraba Darwin contribuyeron a la formación de su teoría de la evolución.

Lo anterior refleja que el análisis de la construcción y transformación representacional constituye un marco teórico que permite dar cuenta, de manera adecuada, de los niveles de comprensión de los conceptos científicos.

$\mathrm{El}$ proceso de construir nuevas representaciones (implícitas y explícitas) o de transformarlas (re-representación) no es sencillo (Flores y Valdez, 2007) pues implica diversos procesos cognitivos (analogías, inferencias, metáforas, patrones, entre otros), así como procesos específicos de enseñanza para las diversas formas de representación (lenguaje, símbolos, ecuaciones, gráficas, etcétera). Por otro lado, no debe perderse de vista que para la construcción de las representaciones científicas los estudiantes utilizan las representaciones procedentes de sus acciones, de los objetos cotidianos que utilizan y los procesos que les sean comunes y no necesariamente con aquellos que corresponden a los que en clase modelan los conceptos y relaciones que les proporcionan los profesores y los textos, y que, en general, no tienen que ver con su entorno, por lo que no los incorporan a sus marcos representacionales.

Las representaciones de los alumnos permiten interpretar los recursos cognitivos que construyen en su entorno escolarizado. Los diversos recursos de que disponga un alumno, en buena medida, determinan su nivel de acción y comprensión y, como se ha descrito, el conjunto o marco de representaciones de que disponga le permitirá dar sentido a lo que se le plantea en la escuela. El uso de las tecnologías digitales, con sus posibilidades de representar procesos, fenómenos, mediciones y gráficas en tiempo real y simulaciones son, en principio, un valioso aporte para la ampliación de los marcos representacionales de los alumnos y, por tanto, para la comprensión de modelos y teorías científicas, por lo que se supone que si los alumnos se encuentran inmersos en un entorno que favorece el uso de múltiples representaciones, tendrán mayores posibilidades de comprensión de los conocimientos científicos.

\section{El contexto: laboratorios de ciencias con TIC}

Los laboratorios equipados con TIC son un espacio que presenta múltiples oportunidades para una enseñanza de las ciencias, donde las representaciones pueden tener un papel predominante, pues en ellos puede conjugarse la experimentación, la medición y 
diversas formas de representar los datos como tablas, gráficas, esquemas, simulaciones y videos, así como compartir las representaciones elaboradas por los alumnos en foros y documentos al acceso de todos en todo momento. El presente estudio se llevó a cabo en escuelas con laboratorios que tienen todas estas posibilidades y que fueron diseñados y construidos por la Universidad Nacional Autónoma de México (UNAM) (Calderón et al., 2016) para sus dos sistemas de bachillerato, la Escuela Nacional Preparatoria (ENP) y el Colegio de Ciencias y Humanidades (CCH), los cuales cuentan con varios de esos laboratorios en cada plantel.

El uso que se da a los laboratorios en cada uno de los planteles y las formas de enseñanza no están estandarizados. Cada escuela y cada profesor define sus formas de trabajo, tanto en organización para asignar laboratorios a profesores y alumnos (algunos son rotativos, otros asignados a un solo profesor por un semestre o un año), como en los procesos didácticos que se llevan a cabo, por lo que los alumnos pueden encontrarse con gran diversidad de formas de enseñanza y de tiempo de uso de estos espacios.

Sobre las principales formas de uso de las TIC en esos laboratorios, el trabajo de Calderón et al. (2016) mostró que, además del interés por incorporar las TIC a sus clases, hay gran diversidad en las competencias digitales de los profesores, y también que hay disciplinas como Biología y Química donde los profesores usan más y de mejor manera las TIC, a diferencia de los cursos de Física, en los que su uso es más limitado.

En cuanto a su potencial para mejorar el aprendizaje de los alumnos, los estudios de Sánchez-Lazo, Gallegos-Cázares y Flores-Camacho (2015) en Química, en el tema de estructura de la materia, y de Cruz-Cisneros, Vega-Murguía, Covarrubias-Martínez, Gallegos-Cázares y Flores-Camacho (2015) en Física, en el tema de cinemática, revelan que esos recursos incorporados en estrategias didácticas orientadas por un marco de aprendizaje en términos de transformación conceptual y representacional, promueve avances significativos en los estudiantes.

\section{Metodología \\ Diseño}

Para cumplir con el propósito del estudio, se dieron tres condiciones: a) trabajar temas de dos materias, para evitar sesgos en el tipo de TIC que se utilizan; b) que dichos temas fueran conocidos previamente por los alumnos, aunque fuera de manera inicial; c) que estos temas hayan sido reportados en la literatura como difíciles para los alumnos y sobre los que se tienen reportadas concepciones alternativas.

Para cumplir con la condición a), en la ENP se analizó el tema de genética, que corresponde a los programas de Biología y, en el CCH el tema de fuerzas y movimiento, que pertenece al programa de Física. Para cumplir la condición b), se seleccionaron temas con los que los estudiantes están familiarizados debido a su contexto cotidiano o escolar. Para cumplir con la condición c), de acuerdo con la literatura, los temas elegidos son complejos y los estudiantes tienen diversas concepciones alternativas (Banet y Ayuso, 2000; Caballero, 2008; Hestenes, Wells y Swackhamer, 1992; Hierrezuelo y Montero, 1989; Iñiguez, 2005; Lewis, Leach y Wood-Robinson, 2000).

Para identificar las representaciones que tienen los estudiantes sobre los temas de genética y fuerzas y movimiento, se diseñaron y aplicaron dos cuestionarios, cada uno correspondiente a cada tema. Dichos instrumentos fueron previamente validados (FloresCamacho, García-Rivera, Báez-Islas y Gallegos-Cázares, 2017).

Se llevaron a cabo dos tipos de análisis. Cuantitativo: análisis de varianza para conocer las diferencias significativas entre las medias de las respuestas de los cuestionarios, y de componentes principales para identificar agrupamientos relevantes entre las respuestas. Cualitativo: análisis de las diferencias de comprensión y formas de representación externa que alcanzan los alumnos en ambos temas. 


\section{Muestra}

La muestra se conformó por dos grupos o submuestras: aquellos que han llevado sus asignaturas de Biología y Física en los laboratorios con TIC (Lab con TIC) y aquellos que no las han cursado en los laboratorios sin TIC (Lab sin TIC). En ambos casos los alumnos cursaban el mismo año escolar; semestres 2016-1 y 2016-2.

En el caso de la ENP, participaron, a petición nuestra y de manera voluntaria, docentes de cuatro planteles, quienes aplicaron el cuestionario de genética a sus alumnos. En el CCH fue la Dirección General la que convocó a profesores de los cinco planteles, quienes aplicaron el cuestionario de fuerzas y movimiento a sus alumnos.

Si bien ambas muestras no son aleatorias, sí son significativas en términos de cumplir con los criterios de uso diferenciado de los recursos tecnológicos y de formas distintas de utilizarlos en las aulas, según el enfoque de cada docente.

Los cuestionarios fueron aplicados a un total de 663 alumnos, de los cuales, 387 corresponden al tema de genética (ENP) y 276 al de fuerzas y movimiento $(\mathrm{CCH})$, como se muestra en la tabla 1.

Tabla 1. Organización de la muestra por grupo y sistema

\begin{tabular}{|c|c|c|c|}
\hline \multicolumn{2}{|c|}{ Lab con TIC } & \multicolumn{2}{c|}{ Lab sin TIC } \\
\hline ENP & CCH & ENP & CCH \\
\hline 170 & 115 & 217 & 161 \\
\hline
\end{tabular}

\section{Instrumentos}

Se elaboraron dos cuestionarios, uno para el tema de genética y otro para el de fuerzas y movimiento. Los ítems se construyeron con la finalidad de que los alumnos pudieran hacer explícitas sus representaciones, a partir de preguntas e instrucciones que promovieran los diversos niveles de explicitación representacional, que van desde la representación pictórica de acciones en el primer nivel, hasta la representación con modelos y teorías en el cuarto nivel, pasando por niveles intermedios descriptivos y simbólicos, de acuerdo con los niveles propuestos por Kozma y Russell (2005). También se consideró que los cuestionarios estuvieran de acuerdo con lo que se denomina Conocimiento Integrado, esto es, "la habilidad para generar ideas científicamente relevantes y uso de teorías, así como de evidencia empírica para conectar ideas en la explicación de fenómenos científicos o justificar proposiciones acerca de un problema científico" (Lee, Liu y Linn, 2011 : 116). Ejemplos de los ítems de ambos cuestionarios se encuentran en el anexo.

La forma en la que se validaron los cuestionarios se encuentra en Flores-Camacho, García-Rivera, Báez-Islas y Gallegos-Cázares (2017). El cuestionario de genética consistió en 13 preguntas, cuatro de las cuales incluyen incisos. En ellas se abordó desde la identificación gráfica de estructuras del material genético en diversos niveles de organización, hasta representaciones y explicaciones de mecanismos de herencia y especialización celular. El cuestionario de fuerzas y movimiento se integró por 22 preguntas que van desde la representación por medio de vectores de variables del movimiento (velocidad, aceleración y fuerza), hasta el uso de la tercera ley de Newton.

Ambos cuestionarios fueron calificados por medio de rúbricas que se desarrollaron con una escala de 0 a 5, adaptando los criterios de Wilson (2005), y considerando los niveles representacionales de 
Kozma y Russell (2005) y del Conocimiento Integrado (Lee, Liu y Linn, 2011), obteniendo así cinco niveles, donde el nivel 0 corresponde a que no hay respuesta, el nivel 1 a ideas y descripciones irrelevantes al problema, el nivel 2 a ideas y representaciones pictóricas inconexas o no precisas, el nivel 3 a ideas y representación relevantes pero sin elaboración, el nivel 4 a ideas y representaciones relevantes y con al menos dos vínculos válidos entre ellas y, el nivel $5 \mathrm{a}$ ideas y representaciones relevantes con más de dos vínculos válidos entre ellas. En la tabla 2 se ejemplifica el uso de las rúbricas.

Tabla 2. Ejemplos del uso de las rúbricas

\begin{tabular}{|c|c|c|}
\hline Nivel & Fuerza y movimiento (pregunta 1.4) & Genética (pregunta 9, incisos a y b) \\
\hline 0 & No contestó. & No contestó. \\
\hline 1 & $\begin{array}{l}\text { Dice que la fuerza lleva mucha velocidad; Los vectores aparecen sin } \\
\text { especificar. }\end{array}$ & $\begin{array}{l}\text { La explicación no hace referencia al material genético ni de cómo se } \\
\text { ve afectado. }\end{array}$ \\
\hline 2 & $\begin{array}{l}\text { Describe que cada vehículo lleva una fuerza y que la fuerza del tráiler } \\
\text { es mayor que la de auto. Los vectores aparecen sin especificar. }\end{array}$ & $\begin{array}{l}\text { Hace mención a que el síndrome de Down es causado por un } \\
\text { problema genético sin hacer referencia del nivel en que se presenta. } \\
\text { Los dibujos que realiza no permiten una interpretación clara del } \\
\text { material genético. }\end{array}$ \\
\hline 3 & $\begin{array}{l}\text { Elabora una gráfica donde la fuerza sobre el auto y el tráiler, decrecen } \\
\text { en el tiempo; dibuja una fuerza de fricción sin especificar. }\end{array}$ & $\begin{array}{l}\text { Menciona que el síndrome de Down es un problema a nivel del } \\
\text { material genético, señalando que es en el ADN, genes o cromosomas, } \\
\text { los que dibuja incompletos. }\end{array}$ \\
\hline 4 & $\begin{array}{l}\text { Describe y grafica que la fuerza sobre el auto alcanza un máximo } \\
\text { en el tiempo de la interacción; la fuerza de fricción actúa en todo } \\
\text { momento; utiliza la tercera ley de Newton de manera incorrecta } \\
\text { indicando que es mayor para un vehículo que para otro. }\end{array}$ & $\begin{array}{l}\text { Reconoce que el síndrome de Down es una alteración cromosómica, } \\
\text { en el que hay un cromosoma de más en el par } 21 \text {. Sus dibujos hacen } \\
\text { referencia a la presencia de un cromosoma de más en el par } 21 .\end{array}$ \\
\hline 5 & $\begin{array}{l}\text { Además de las ideas presentes en el nivel } 4 \text {, utiliza la tercera ley de } \\
\text { Newton para explicar que ambos objetos reciben la misma fuerza, } \\
\text { pero en sentido contrario. }\end{array}$ & $\begin{array}{l}\text { Además de las ideas presentes en el nivel } 4 \text {, describe que es durante } \\
\text { el proceso de meiosis donde no hay una correcta separación de los } \\
\text { cromosomas homólogos del par } 21 .\end{array}$ \\
\hline
\end{tabular}

Nota. Pregunta 1.4. En la siguiente figura (gráfica fuerza vs tiempo) correspondiente a esta situación (colisión entre dos vehículos), dibuja la fuerza o las fuerzas presentes en el momento del choque entre ambos vehículos. También, explica qué representa lo que has dibujado. Pregunta 9. Alicia tiene miedo de que el bebé nazca con síndrome de Down, ayúdala a comprender mejor esta situación, respondiendo si: a) Esto se debe a que hay una alteración genética en la información del bebé. ¿Esto es cierto? ¿Por qué? b) Si consideras que es cierto, haz un dibujo con el que expliques claramente a Alicia dónde se presenta esta alteración y cómo es posible ilustrarla (no olvides describir por escrito lo que representas en tu dibujo).

\section{Resultados}

Los resultados se presentan por el tipo de análisis empleado, primero se describe el análisis cuantitativo para cada tema y, posteriormente, el cualitativo. En el análisis cuantitativo se presenta un tratamiento diferenciado de los datos debido a que en el tema de genética las diferencias entre los grupos con TIC y sin TIC son más contundentes a favor de los alumnos que trabajaron en los laboratorios con TIC, mientras que, para el caso de física, las diferencias son menores, si bien significativas en aspectos clave, como se detallará en este apartado. 


\section{Análisis cuantitativo}

\section{Tema: genética}

$\mathrm{El}$ análisis de varianza (ANOVA-SPSS) mostró diferencias significativas en todas las preguntas entre ambos grupos, indicando que los alumnos que cursaron la materia de Biología en los laboratorios con TIC responden mejor el cuestionario que los que la llevaron en los laboratorios sin TIC. La tabla 3 presenta los datos obtenidos para un nivel de significatividad $p \leq 0.05$.

La pregunta 1 obtuvo el valor más bajo de $F(11.3)$, las demás preguntas mostraron valores superiores a 24, y la pregunta 5 alcanzó el valor más alto de $F$ (93.3). Las preguntas 1 y 5 requerían explicaciones conceptuales. La pregunta 1 hacía referencia a la herencia de características de padres a hijos de manera general, y los resultados muestran que las respuestas de los alumnos de ambos grupos fueron similares. La pregunta 5 solicitaba a los alumnos que explicaran las diferencias entre la información de las células de uno de los progenitores, los alumnos del grupo Lab con TIC dieron respuestas que se ubicaron entre los niveles 2 a 5 de la rúbrica, y los alumnos del grupo Lab sin TIC, respondieron entre los niveles 0 y 5 . La diferencia de más de un punto en la media entre los grupos para esta pregunta puede relacionarse con la posibilidad de que los estudiantes que contaron con tecnología tuvieron más oportunidades para observar diferentes tipos celulares y su material genético.

Tabla 3. Resultados del análisis de varianza

\begin{tabular}{|c|c|c|c|c|}
\hline \multirow{2}{*}{ Pregunta } & \multicolumn{2}{|c|}{ Media } & \multirow{2}{*}{$\boldsymbol{F}$} & \multirow{2}{*}{$p$} \\
\hline & Lab con TIC & Labsin TIC & & \\
\hline 1 & 3.05 & 2.80 & 11.369 & 0.001 \\
\hline 2 & 3.61 & 3.18 & 24.502 & 0.000 \\
\hline 3 & 3.17 & 2.47 & 30.427 & 0.000 \\
\hline 4 & 3.07 & 2.27 & 39.673 & 0.000 \\
\hline 5 & 3.38 & 2.42 & 93.339 & 0.000 \\
\hline 6 & 2.98 & 2.11 & 60.439 & 0.000 \\
\hline $7 a, b$ & 3.04 & 2.32 & 27.774 & 0.000 \\
\hline $7 c$ & 2.98 & 2.14 & 42.532 & 0.000 \\
\hline $7 d$ & 3.24 & 2.18 & 58.706 & 0.000 \\
\hline $7 \mathrm{e}, \mathrm{f}, \mathrm{g}$ & 3.45 & 2.79 & 57.191 & 0.000 \\
\hline $8 \mathrm{a}, \mathrm{b}$ & 3.14 & 2.32 & 55.136 & 0.000 \\
\hline $8 c$ & 3.25 & 2.37 & 75.651 & 0.000 \\
\hline $9 \mathrm{a}, \mathrm{b}$ & 3.71 & 2.99 & 73.042 & 0.000 \\
\hline $9 c$ & 3.38 & 2.53 & 55.66 & 0.000 \\
\hline 10 & 3.32 & 2.82 & 27.831 & 0.000 \\
\hline 11 & 3.32 & 2.55 & 56.914 & 0.000 \\
\hline 12 & 2.91 & 2.28 & 40.889 & 0.000 \\
\hline $13 a, b, c, d, e, f, g$ & 3.99 & 3.09 & 49.266 & 0.000 \\
\hline
\end{tabular}

Nota. Las preguntas 1, 2, 5, 7 defg, 8abc y 12 buscan explicaciones que den cuenta de cuál es la información que los progenitores heredan a la descendencia, y cuáles son los mecanismos que explican la variabilidad genética en la expresión de las características. Las preguntas 3, 4, 6, 7abc y 13abcdefg indagan, mediante el uso de representaciones externas, la comprensión de los alumnos sobre cómo se organiza la información genética, qué información genética está presente en una célula haploide y en una célula diploide, y cómo se relaciona esta información genética en la expresión de una característica (relaciones genotipo-fenotipo). Las preguntas $9 a b c, 10$ y 11 están referidas a explicaciones sobre las alteraciones genéticas. 
Para tener una idea de las diferencias típicas en las respuestas de los alumnos en los distintos niveles de la rúbrica, se presentan dos fragmentos, de dos alumnos, correspondientes a la pregunta 9 incisos a y b, que dice: "Alicia tiene miedo de que el bebé nazca con síndrome de Down, ayúdala a comprender un poco mejor esta situación, respondiendo a sus dudas: a) Esto se debe a que hay una alteración genética en la información del bebé. ¿Esto es cierto? ¿Por qué?”

1. Alumno del grupo Lab sin TIC; obtuvo el nivel 3 en la rúbrica (para el inciso a): "Sí, en el síndrome de Down ocurre que en el cromosoma 21 no hay una separación (rompimiento), éste queda unido alterando así la información".

2. Alumno del grupo Lab con TIC; obtuvo el nivel 3 en la rúbrica (para el inciso a): "Sí es cierto. El síndrome de Down es una alteración en la que se tiene un cromosoma de más en el par No. 21 por lo tanto es una alteración genómica".

Puede notarse que, hasta este momento, en ambas respuestas los alumnos logran una comprensión del tema (Integración del Conocimiento) que los ubica en un nivel 3 debido a que reconocen que el síndrome de Down es una alteración genética que ocurre en el cromosoma 21, sin dar alguna explicación que dé cuenta de por qué ocurre. Sin embargo, al analizar el inciso b, es posible notar diferencias en su construcción representacional:

"b) Si consideras que es cierto, haz un dibujo con el que expliques claramente a Alicia dónde se presenta esta alteración y cómo es posible ilustrarla (no olvides describir por escrito lo que representas en tu dibujo)".

1. Mismo alumno del grupo Lab sin TIC; obtuvo el nivel 3 en la rúbrica (para el inciso b) (véase figura 1).

Figura 1. Respuesta pregunta 9 b) Lab sin TIC

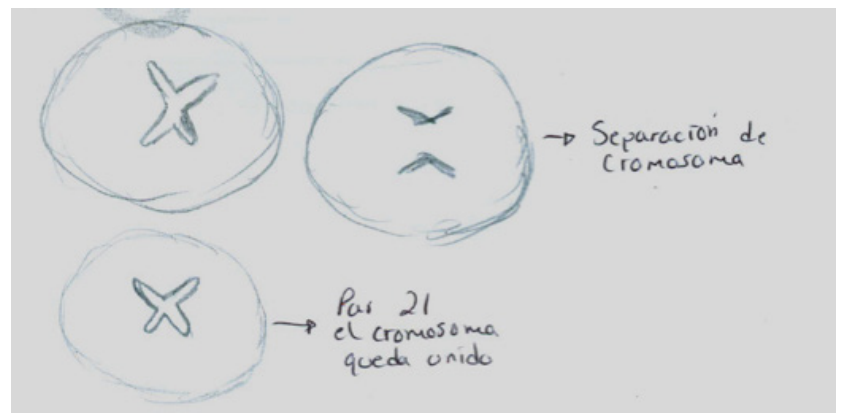

En esta representación gráfica podemos observar que el alumno representa lo descrito en el inciso a), donde identifica que el síndrome ocurre debido a que no ocurre una separación correcta de las cromátides del cromosoma en el par 21; sin embargo, no explicita en qué momento ocurre la separación cromosómica, cuántos pares debe tener la célula del individuo o cómo se representa el cariotipo del individuo con esta alteración.

2. Mismo alumno del grupo Lab con TIC; obtuvo el nivel 5 en la rúbrica (para el inciso b) (véase figura 2).

\section{Figura 2. Respuesta pregunta 9 b) Lab con TIC}

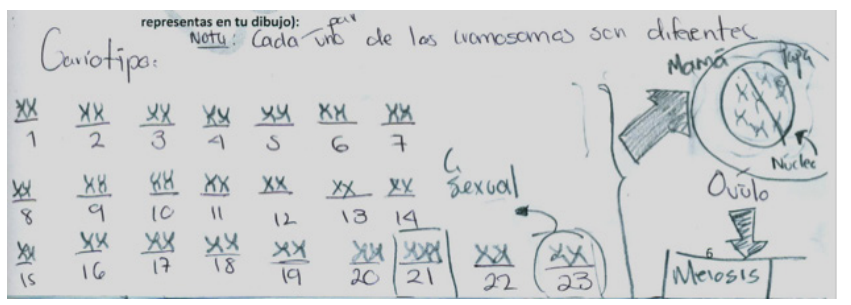


En este caso podemos notar que la representación realizada hace explícito el proceso y muestra la comprensión que alcanza el alumno con respecto a que se requiere realizar un cariotipo para conocer dónde se encuentra la alteración cromosómica, que la información genética está conformada por 23 pares de cromosomas y el proceso en el cual ocurre la alteración genética es en una célula sexual durante la meiosis.

Para agrupar el tipo de respuestas en función de las características sobre el tipo de representaciones que usan ambos grupos, se llevó a cabo un análisis de componentes principales, que se muestra en la tabla 4.

Tabla 4. Resultados del análisis de componentes principales

\begin{tabular}{|l|c|c|c|c|}
\hline \multirow{2}{*}{ Pregunta } & \multicolumn{4}{c|}{ Componente } \\
\cline { 2 - 5 } & $\mathbf{1}$ & $\mathbf{2}$ & $\mathbf{3}$ & $\mathbf{4}$ \\
\hline Pregunta 1 & 0.392 & 0.060 & 0.081 & 0.697 \\
\hline Pregunta 2 & 0.329 & 0.007 & 0.078 & 0.127 \\
\hline Pregunta 3 & 0.575 & 0.435 & 0.547 & -0.025 \\
\hline Pregunta 4 & 0.630 & 0.426 & 0.491 & -0.114 \\
\hline Pregunta 5 & 0.609 & -0.017 & 0.025 & 0.040 \\
\hline Pregunta 6 & 0.599 & 0.364 & 0.207 & 0.122 \\
\hline Pregunta 7, a, b & 0.563 & 0.542 & -0.496 & -0.148 \\
\hline Pregunta 7 c & 0.569 & 0.465 & -0.567 & -0.103 \\
\hline Pregunta 7 d & 0.642 & -0.085 & 0.006 & -0.182 \\
\hline Pregunta 7 e, f, g & 0.651 & -0.115 & -0.269 & 0.066 \\
\hline Pregunta 8, a y b & 0.706 & -0.217 & -0.109 & 0.078 \\
\hline Pregunta 8 c & 0.650 & -0.322 & -0.039 & 0.133 \\
\hline Pregunta 9 a, y b & 0.612 & -0.293 & 0.084 & 0.100 \\
\hline Pregunta 9 c & 0.577 & -0.420 & 0.093 & -0.077 \\
\hline Pregunta 10 & 0.463 & -0.510 & 0.073 & -0.277 \\
\hline Pregunta 11 & 0.638 & -0.207 & -0.006 & -0.354 \\
\hline Pregunta 12 & 0.534 & -0.143 & -0.244 & 0.386 \\
\hline Pregunta 13 a, b, c, d, e, f, g & 0.705 & 0.051 & 0.070 & -0.172 \\
\hline
\end{tabular}

Las preguntas que agrupa el primer componente (tabla 4) corresponden a preguntas orientadas a que los alumnos expliciten qué información genética está presente en diferentes tipos celulares y cómo se da la expresión de caracteres en el fenotipo (5; 6; 7d; 8a, b; 8c; 12), también agrupa preguntas orientadas a proporcionar explicaciones sobre la expresión de los síndromes (9a, b; 9c; 10, 11) y sobre las alteraciones genéticas (8a, b, y 8c). Cabe resaltar que las preguntas que demandaban una representación gráfica o simbólica del material genético y de procesos hereditarios (7e, f, g; 9a, b; 13a, b, c, $\mathrm{d}, \mathrm{e}, \mathrm{f}, \mathrm{g})$, también quedan dentro de este componente.
El segundo componente agrupa dos preguntas (7a, b y $7 \mathrm{c})$, referidas a las descripciones que requieren de utilizar el modelo de herencia mendeliana. En este caso se reducen las diferencias en los valores de las medias. El tercer componente agrupa dos preguntas ligadas (3 y 4) que solicitaban una representación gráfica de cómo se encuentra organizada la información genética dentro de las células sexuales. El cuarto componente comprende sólo la primera pregunta, sobre generalidades de la herencia de características de padres a hijos y que, como se apuntó, tiene el valor más bajo en la prueba de varianza. 
El primer componente, que agrupa la mayoría de las preguntas, nos muestra que las diferencias relevantes entre ambos grupos están en el nivel de comprensión y de representación entre ellas, pues en todas esas preguntas se tiene que las diferencias en las medias son las mayores y significativas (ver tabla 3), indicando que los alumnos que han participado en los Lab con TIC, muestran mejor comprensión de los procesos genéticos y de sus formas de representarlos.

\section{Tema: fuerzas y movimiento}

A diferencia del tema de genética, en el caso de fuerzas y movimiento los resultados no son tan contundentes, pues la mayoría de las preguntas no presentan diferencias significativas. Sin embargo, como se verá en el análisis, los datos son relevantes en términos de la comprensión del proceso físico.

El análisis de varianza (ANOVA-SPSS) muestra diferencias significativas en 6 de las 17 preguntas. La tabla 5 muestra los resultados para una significatividad de $\mathrm{p} \leq 0.05$ (se presentan sólo los resultados con diferencia significativa).

Tabla 5. Resultados del análisis de varianza

\begin{tabular}{|c|c|c|c|c|}
\hline \multirow{2}{*}{ Pregunta } & \multicolumn{2}{|c|}{ Media } & \multirow{2}{*}{$F$} & \multirow{2}{*}{$p$} \\
\hline & Lab con TIC & Lab sin TIC & & \\
\hline 1.2 & 2.11 & 1.99 & 29.797 & 0.026 \\
\hline 1.5 & 2.02 & 1.73 & 9.683 & 0.019 \\
\hline 1.7 & 2.48 & 2.22 & 6.572 & 0.011 \\
\hline 1.9 & 2.17 & 1.85 & 2.532 & 0.011 \\
\hline 1.11 & 2.28 & 2.04 & 2.818 & 0.031 \\
\hline 1.12 & 2.08 & 2.48 & 17.892 & 0.005 \\
\hline
\end{tabular}

Nota. La pregunta 1.2 indaga sobre los efectos (aceleración) de los conductores en la colisión (vehículos diferentes), la pregunta 1.5 pide una representación gráfica de fuerza contra tiempo durante la colisión, la pregunta 1.7 es sobre procesos de medición de fuerzas y movimiento con distintos medios, la pregunta 1.9 es directa sobre la comprensión de la tercera ley de Newton y las preguntas 1.11 y 1.12 indagan también sobre las fuerzas presentes y los efectos en los conductores pero con condiciones de vehículos iguales.

Los valores obtenidos en las medias dan cuenta de que los ítems 1.2, 1.5, 1.7, 1.9 y 1.11 del grupo Lab con TIC, obtienen mayores puntuaciones en las respuestas de la rúbrica, principalmente en la pregunta 1.2. Por su parte, en el grupo Lab sin TIC, la pregunta 1.12 obtiene una media mayor que el grupo Lab con TIC. En cuanto al tipo de demanda de los ítems, la pregunta 1.2 implica la descripción de los efectos que sienten los conductores de los vehículos que colisionan, de la que se espera una respuesta relacionada con la aceleración; la pregunta 1.5 implica la representación gráfica del comportamiento de la fuerza durante el choque; la pregunta 1.7, concierne a cómo medir la fuerza con distintos medios de registro; la pregunta 1.9 aborda la aplicación de la tercera ley de Newton; y la pregunta 1.11 es semejante a la 1.2 cambiando las condiciones de los vehículos de diferentes a iguales.

A pesar de que son pocas las preguntas en que hay diferencias significativas entre los grupos Lab con TIC y Lab sin TIC, en ellas se encuentran los aspectos sobre la comprensión del proceso en términos de la aplicación de la tercera ley de Newton, la descripción del comportamiento de la fuerza en el tiempo, y los efectos en términos de aceleración. 
La pregunta 1.12 en la que el grupo Lab sin TIC, aventaja al grupo Lab con TIC, se refiere a la representación vectorial de las fuerzas y es la que puede encontrarse usualmente en los libros de texto. El resto de las preguntas, que no fueron significativamente diferentes, involucran otros aspectos como velocidad, fuerza de gravedad, inercia y cómo representarlas vectorialmente y en el tiempo.

Para ejemplificar las diferencias en el tipo de respuestas que se obtienen en los diferentes niveles de la rúbrica, se presentan dos respuestas típicas para la pregunta 1.9 que dice: "Si alguien te preguntara si en esta situación vale o se aplica la tercera ley de Newton, ¿qué le responderías y cómo le explicarías tu respuesta? Si lo requieres, para apoyar tu respuesta realiza uno o varios dibujos de tu explicación".

3. Alumno del grupo Lab sin TIC; obtuvo el nivel 3 en la rúbrica: 'A toda acción corresponde una reacción de igual magnitud, pero en sentido contrario'. El auto y el tráiler chocaron, pero el impacto hizo que regresaran y se produjo un rebote" (véase figura 3).

Este alumno enuncia las fuerzas de acción y reacción, pero no logra explicar cómo se aplica a cada vehículo e introduce factores ajenos a la pregunta. Su dibujo es congruente con su explicación y obtiene el mismo nivel.

\section{Figura 3. Respuesta gráfica a pregunta 1.9 (alumno Lab sin TIC)}

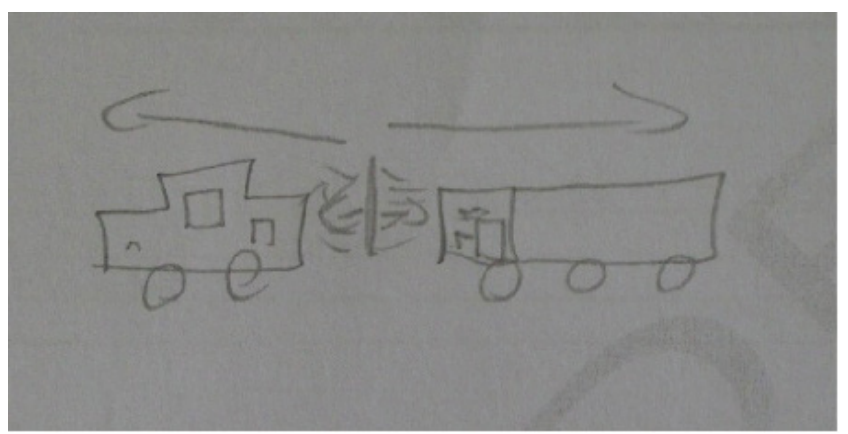

4. Alumno del grupo Lab con TIC; obtuvo el nivel 5 en la rúbrica: "Le respondería que sí, ya que un objeto (el auto) está aplicando una fuerza sobre el otro (el tráiler) de igual magnitud, pero en sentido contrario" (véase figura4).

Este alumno describe de manera correcta la aplicación de la tercera ley de Newton, sin embargo, como ocurrió con todos los alumnos de la muestra de este tema, no es consistente con el punto de aplicación de las fuerzas y en su dibujo, solo alcanza el nivel 3 de la rúbrica.

Debido a que son pocos los ítems con diferencias significativas, el método de componentes principales no aportaría mayores elementos para el análisis de los resultados.

Figura 4. Respuesta gráfica a la pregunta 1.9 (Alumno Lab con TIC)

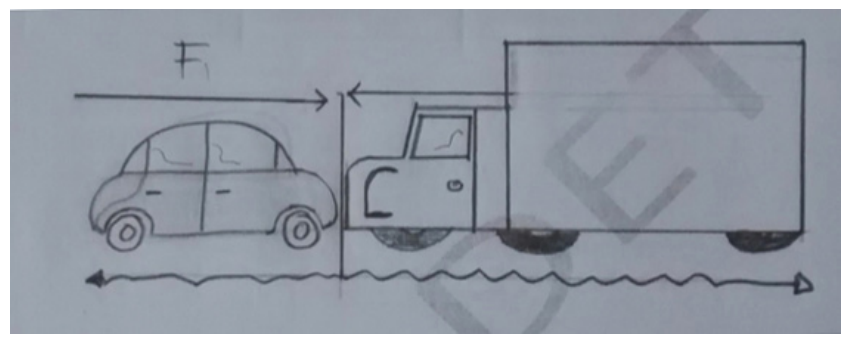




\section{Discusión}

Los resultados muestran que la presencia de diversos medios de representación tiene efectos positivos en los alumnos, en cuanto a su comprensión y posibilidades de representar los conocimientos científicos. Muy favorables para el caso de Biología, y menos relevantes para Física. Sin embargo, se mantiene abierta la pregunta sobre las diferencias cualitativas que muestran los alumnos del grupo Lab con TIC, sobre cómo explican y usan los conceptos, así como la precisión y claridad con la que los representan.

\section{Análisis cualitativo}

\section{Tema: genética}

Uno de los aspectos básicos para comprender este tema es el reconocimiento de la representación gráfica y simbólica del material genético en diversos niveles de organización y, por tanto, poderlos describir a través de distintos niveles representacionales. Esto se hace evidente en la pregunta 3 en la que se solicita que se realice un dibujo que muestre y explique cómo se encuentra la información genética en el espermatozoide. En seguida se muestra una comparación de dos respuestas que obtuvieron el mismo puntaje en la rúbrica (nivel 3). La figura 5 muestra los dibujos y descripciones de un alumno del grupo Lab con TIC (a) y uno del grupo Lab sin TIC (b). Puede notarse que las explicaciones son muy similares, pues en ambos casos el alumno identifica que existen diferentes niveles de organización, los cuales enuncia. En cuanto a sus representaciones, podemos observar que existen diferencias sutiles entre los ejemplos, lo cual indica que, a pesar de encontrarse en un mismo nivel en la rúbrica, las representaciones de los alumnos de Lab con TIC pueden ser más completas; se observa que en ambos casos su representación del espermatozoide es muy cercana; sin embargo, el alumno Lab con TIC (a) representa el cromosoma que se encuentra conformado por ADN compactado, el cual a su vez se encuentra formado por nucleótidos y en su dibujo enfatiza la unión de estos para la formación de la cadena de ADN, mientras que el alumno de Lab sin TIC (b), aunque menciona también las uniones y otros niveles de organización, solo representa el ADN.

Figura 5. Ejemplos de las representaciones de los grupos: (a) Lab con TIC y (b) Lab sin TIC.

(a)

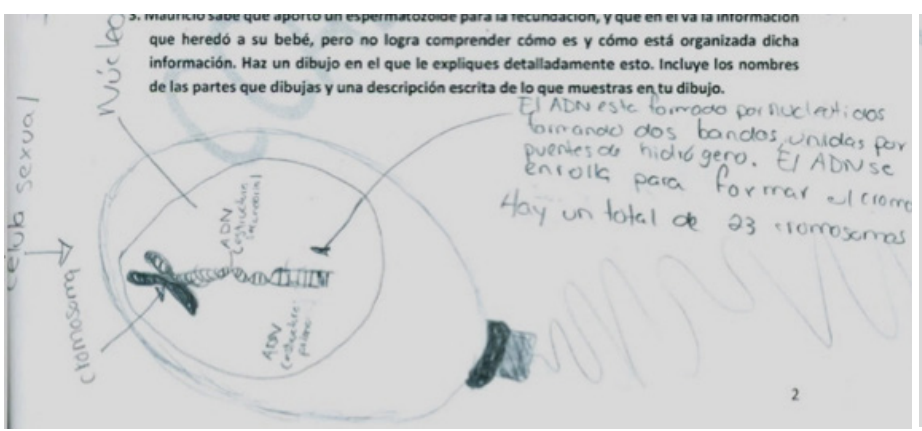

Acerca de la comprensión de los procesos en las células, el grupo Lab sin TIC obtiene la media más baja $(M=2.11)$ en la pregunta 6 , que está orientada a conocer si han comprendido que, en todas las células, y no solo en las sexuales, hay información genética y cómo ésta es expresada a partir de diferentes mecanismos. Los resultados, en general, indican que (b)

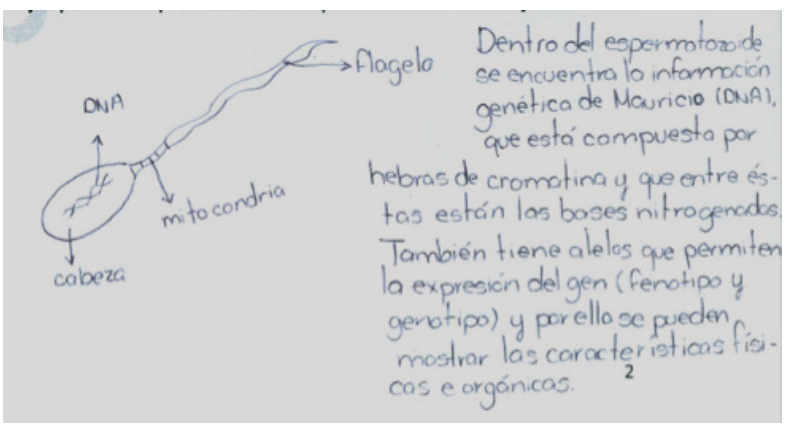

los alumnos que no contaron con apoyo de tecnología tuvieron, en principio, mayores dificultades para representar gráficamente y explicar los conceptos y estructuras implicadas en preguntas como la 6, que buscaba que los estudiantes fueran más allá de pensar en las funciones de las células y reconocieran aspectos de especialización celular. 
Lo anterior se ve ilustrado en las representaciones que alumnos del grupo Lab con TIC y Lab sin TIC hacen para responder parte de la pregunta 6 . En la figura 6 caso (a), que obtuvo un nivel 3 de la rúbrica, se muestra la representación de un alumno del grupo Lab con TIC y el caso (b) muestra la respuesta de un alumno del grupo Lab sin TIC. Puede apreciarse que el alumno del caso (a) indica que en el espermatozoide hay 23 cromosomas y es una célula 1n, distinguiéndola de la célula de la piel (somática), en la que indica hay 46 cromosomas y es 2n, lo que no ocurre con el estudiante del caso (b), que alcanzó un nivel 2 en la rúbrica (muy pocos alumnos de este grupo alcanzan el nivel 3), pues piensa que sólo hay información genética (y sólo cromosomas sexuales) en el espermatozoide y que la célula somática no tiene información genética, pues en ella solo reconoce proteínas.

\section{Figura 6. Representaciones de la información que hay dentro de un espermatozoide (izquierda) y una célula de la piel (derecha). Caso (a) alumno del grupo Lab con TIC, y caso (b) alumno del grupo Lab sin TIC.}

(a)
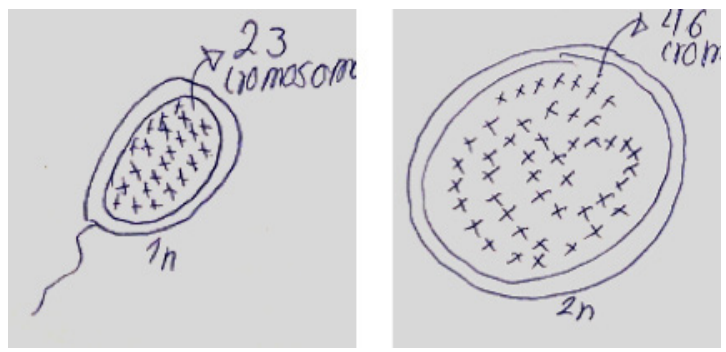

La comprensión de la representación de la figura 6 , se ve fortalecida por la explicación que cada alumno da a sus dibujos. Para el caso (a) el alumno describe: "Todas las células somáticas poseen la misma información genética, lo mismo sucede con las células sexuales o gametos, en este caso varía únicamente el juego cromosómico (2n y n respectivamente)".

En su explicación, reconoce que la información de todas las células es la misma, independientemente de su función, y menciona que las diferencias entre células somáticas y sexuales son debidas al número de cromosomas de cada una. Su respuesta corresponde con los dibujos que hizo.

Para el caso (b) el alumno describe: "Sí, porque cada célula del cuerpo tiene una cierta función, o sea no todas pueden hacer lo de la otra. Bueno más bien tienen la misma información ya que es individuo, pero como ya dije cada una tiene una función".

En su explicación logra reconocer que la (b)
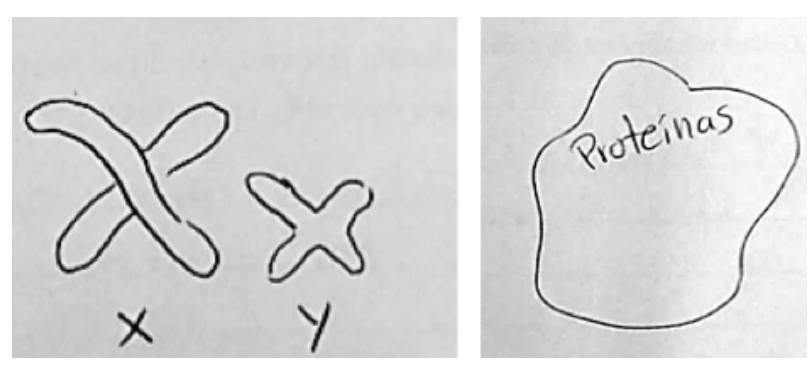

información de todas las células es la misma, sin embargo, no hace referencia a similitudes o diferencias entre células somáticas y sexuales. Su respuesta no está ligada a las representaciones gráficas que realizó, y muestra que no ha logrado una comprensión completa de lo que se pregunta.

\section{Tema: fuerzas y movimiento}

De manera equivalente a la descripción para el caso de Biología, en Física también es posible notar las diferencias cualitativas en la comprensión y representación que alcanzan los alumnos del grupo Lab con TIC, con respecto a los del grupo Lab sin TIC. Por ejemplo, en cuanto a posibilidades de representación gráfica, se muestran las respuestas a la pregunta 1.5, "Suponiendo que el choque ocurrió durante tres segundos, desde el momento del impacto hasta que ambos vehículos quedaron en reposo, dibuja en la siguiente gráfica la fuerza (o las fuerzas) sobre el auto 
compacto. También, explica por qué se comporta así esa fuerza (o fuerzas) conforme a lo que dibujaste". Un primer aspecto es que ningún alumno del grupo Lab sin TIC tiene una respuesta que se acerque a describir el comportamiento de la fuerza, mientras que el 6.4\% del grupo Lab con TIC, representa que, justo en el momento del impacto, la fuerza alcanza su máximo valor y que antes y después del impacto no hay fuerza. La figura 7 ilustra las diferencias de representación.

\section{Figura 7. Caso (a) alumno del grupo Lab con TIC. Caso (b) Lab sin TIC.}

(a)

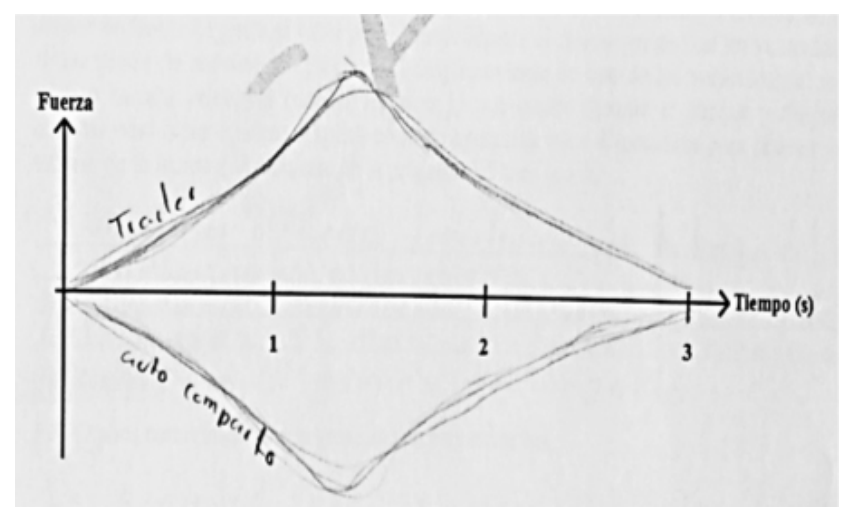

La figura del caso (a) es una representación aproximada a una descripción física correcta (representa las fuerzas en ambos vehículos). En el caso (b), el alumno del grupo Lab sin TIC, muestra el comportamiento de la fuerza sobre un vehículo, pero en éste permanece una fuerza después de la colisión.

En cuanto a la comprensión que muestran los alumnos para interpretar el comportamiento de las fuerzas y otras variables del movimiento en ese proceso, los alumnos del grupo Lab con TIC presentan una ligera mejora en cuanto a sus explicaciones. Ejemplos de respuestas típicas de ambos grupos a la pregunta 1.2 acerca de los efectos que sienten los conductores de ambos vehículos son:

Alumno del grupo Lab con TIC; obtuvo el nivel 4 en la rúbrica: "El conductor del auto compacto. Porque es el que adquiere mayor aceleración, entonces en el choque será quien lo sienta más”.

Alumno del grupo Lab sin TIC; obtuvo el nivel 3 en la rúbrica (no hay alumnos de este grupo que alcancen el nivel 4 para esta pregunta): "El conductor (b)

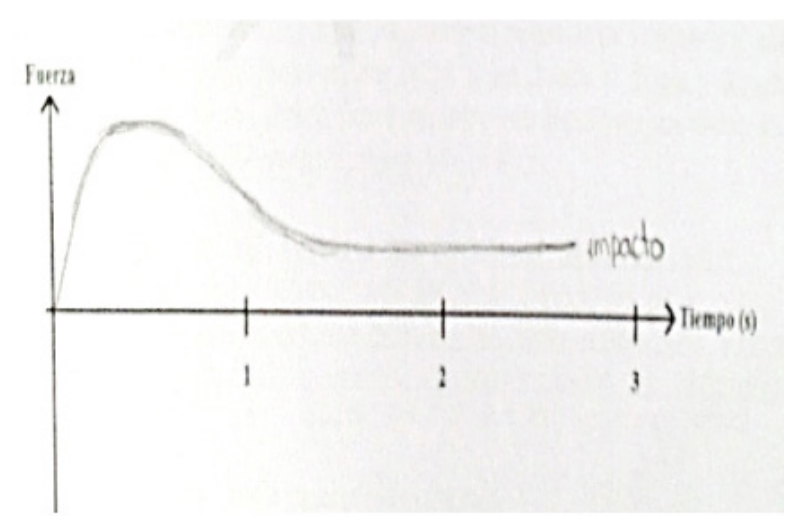

del auto compacto, ya que el tamaño del auto es menor, por lo tanto, el impacto que recibe es mayor que el que recibe el tráiler, por lo tanto, sufre más daño el conductor del automóvil."

El alumno del grupo Lab con TIC describe lo que siente el conductor en términos de la aceleración, correspondiente a la fuerza en el impacto, mientras que el alumno del grupo Lab sin TIC lo atribuye al tamaño de los vehículos.

\section{Conclusiones}

Los resultados descritos dan indicios de que la introducción en las escuelas de laboratorios de ciencias con una gran variedad de recursos tecnológicos tiene influencia positiva en la comprensión y posibilidad de representación que logran los alumnos, como se muestra en que más alumnos que estuvieron en los laboratorios con TIC obtuvieron niveles más altos en la rúbrica y mostraron representaciones esquemáticas y gráficas más completas o más apegadas a los procesos que describen, si bien esto no ocurre por 
igual para todas las materias, y con la consideración de que en un análisis más concluyente habrá que tomar en cuenta, qué tipo de procesos didácticos utilizan los docentes, así como describir las formas y frecuencia de uso de esos recursos. Por otra parte, el estudio no lleva a cabo ningún control sobre la muestra, ni sobre los profesores (por ejemplo, indicaciones sobre procesos de enseñanza, usos adecuados de la tecnología o cualquier otro factor que pudiera alterar lo que cotidiana y espontáneamente ocurre en las clases). Si bien hay que tomar en cuenta que los profesores que permitieron aplicar los cuestionarios a los alumnos no se seleccionaron aleatoriamente, pues hubo participación voluntaria, la condición de ninguna intervención, se cumplió en todos los casos.

Este estudio tiene entre sus hipótesis que un factor relevante de contar con las TIC en los laboratorios es la posibilidad que tienen los alumnos de interaccionar con múltiples representaciones. Esto parece ser más relevante en el caso de Biología que en Física, lo que es congruente en lo reportado por Calderón et al. (2016), donde se muestra que los profesores de Biología y Química usan más y mejor los recursos de los laboratorios, pues los resultados indican claramente que los alumnos del grupo Lab con TIC evidencian una mejor comprensión, identificación y posibilidad de representación de los conceptos y procesos biológicos, lo que sugiere que, en sus clases, esos recursos son más utilizados y, posiblemente, son empleados de mejor forma por los docentes; es decir, con mayor énfasis en las formas de representación externa y sus posibilidades para apoyar la comprensión de los conocimientos científicos.

Este estudio sólo puede considerarse como una primera incursión al análisis de los efectos que la modificación del contexto para la enseñanza de las ciencias en las escuelas puede tener, sobre todo, un contexto en el que hay una profusa incorporación de las TIC que, además de los aspectos de obtención de información y visualización, incluyen también procesos de medición y observación experimental. Sin embargo, no pretende ni debe inferirse afirmación alguna de que la tecnología por sí sola hace alguna diferencia, sino que implica ventajas cuando es empleada por los profesores de manera intencional, aunque haya diferencias en los enfoques didácticos desde los cuales se emplean.

A partir de este estudio exploratorio surgen nuevas interrogantes para lograr una mejor comprensión de los efectos y problemáticas que la incorporación de las TIC como recursos de representación pueden lograr mejoras en el aprendizaje. Algunas de esas interrogantes son:

¿Cómo afectan las formas de enseñanza y de interacción de los profesores con sus alumnos, cuando en las escuelas se introducen gran cantidad de tecnologías digitales? Esta pregunta tiene su origen en que, si se identifica que hay diferencias relevantes en la práctica docente entre ambos grupos, permitiría explicar, al menos en parte, los resultados obtenidos.

¿Cuáles son las razones de las diferencias en los resultados entre los de alumnos de las materias de Biología y Física? ¿tienen que ver con la estructura de la disciplina?

¿El logro observado con la introducción de las TIC, es independiente de las concepciones de aprendizaje de los profesores?

Agradecimientos. Este proyecto fue apoyado por el programa PAPIME-PE302315. Los autores agradecen a Nancy Montes Calva y a Manuel Cruz Cisneros por sus aportaciones al proyecto. 


\section{Referencias}

Ainsworth, Shaaron (2006), "DeFT: a conceptual framework for considering learning with multiple representations", Learning and Instruction, vol. 16, pp. 183-198.

Al-Balushi, Sulaiman M., Ali S. Al-Musawi, Abdullah K. Ambusaidi y Fatemah H. Al-Hajri (2017), "The effectiveness of interacting with scientific animations in chemistry using mobile devices on grade 12 students' spatial ability and scientific reasoning skills", fournal of Science Education E̊ Technology, vol. 26, núm. 1, pp. 70-81, <https://doi.org/10.1007/s10956-016-9652-2>.

Banet, Enrique y Enrique Ayuso (2000), "Teaching genetics at secondary school: a strategy for teaching about the location of inheritance information", Science Education, vol. 84, pp. 313-351.

Caballero, Manuela (2008), "Algunas ideas del alumnado de secundaria sobre conceptos básicos de genética", Enseñanza de las Ciencias, vol. 26, núm. 2, pp. 227-243.

Calderón-Canales, Elena, Fernando Flores-Camacho, Leticia Gallegos-Cázares, Gustavo de la Cruz-Martínez, Jesús Ramírez-Ortega y Ricardo Castañeda-Martínez (2016), "Laboratorios de ciencias en el bachillerato: tecnologías digitales y adaptación docente", Revista Apertura, vol. 8, núm. 1, pp. 1-17.

Chandra, Vinesh y Margaret Lloyd (2008), "The methodological nettle: ICT and student achievement", British Journal of Educational Technology, vol. 39, núm. 6, pp. 1087-1098.

Cook, Michelle Patrick (2006), "Visual representations in science education: the influence of prior knowledge and cognitive load theory on instructional design principles", Science Education, vol. 90, pp. 1073-1091, <DOI: 10.1002 /sce.20164>.

Cruz-Cisneros, Jesús, Eduardo Vega-Murguía, Héctor Covarrubias-Martínez, Leticia Gallegos-Cázares y Fernando Flores-Camacho (2015), "Movimiento debido a la gravedad. Una experiencia multirepresentacional en los nuevos laboratorios del bachillerato de la UNAM", Latin American Fournal of
Physics Education, vol. 9, núm. 1, pp. 1403-1-1403-8.

Díaz-Barriga, Ángel (2013), "TIC en el trabajo del aula. Impacto en la planeación didáctica", Revista Iberoamericana de Educación Superior (RIES), vol. 4, núm. 10, pp. 3-21.

Eilam, Billie y John K. Gilbert (2014), Science Teachers' use of visual representations, Switzerland, Springer.

Flores, Fernando y Ricardo Valdez (2007), "Enfoques epistemológicos y cambios representacionales y conceptuales", en J. I. Pozo y F. Flores (eds.), Cambio conceptual y representacional en el aprendizaje y la enseñanza de la ciencia, Madrid, Antonio Machado Libros, pp. 21-36.

Flores-Camacho, Fernando, Beatriz. E. García-Rivera, Araceli Báez-Islas y Leticia Gallegos-Cázares (2017), "Diseño y validación de un instrumento para analizar las representaciones externas de estudiantes de bachillerato sobre genética", Revista Iberoamericana de Evaluación Educativa (RIES), vol. 10, núm. 2, pp. 151169.

Gale, Jessica, Stefanie Wind, Jayma Koval, Joseph Dagosta, Mike Ryan y Marion Usselman (2016), "Simulation-based performance assessment: an innovative approach to exploring understanding of physical science concepts", International fournal of Science Education, vol. 38, núm. 14, pp. 2284-2302, <DOI: 10.1080/09500693.2016.1236298>.

Geelan, David R. y Xinxin Fan (2013), "Teachers using interactive simulations to scaffold inquiry instruction in physical science education", en Billie Eilam y John K. Gilbert (eds.), Science teacher's use of visual representations, Switzerland, Springer International Publishing, pp. 249-270.

Haglund, Jesper, Fredrik Jeppsson y Konrad Schönborn (2016), "Taking on the heat- a narrative account of how infrared cameras invite instant inquiry", Research in Science Education, vol. 46, núm. 5, pp. 685-713.

Hestenes, David, Malcolm Wells y Gregg Swackhamer (1992), "Force concept inventory", The Physics Teacher, vol. 30, núm. 3, pp. 141-158. 
Hierrezuelo, Moreno José y Antonio Montero Moreno (1989), La ciencia de los alumnos: su utilización en la didáctica de la fisica y la química, España, Editorial Laia/Ministerio de Educación y Ciencia.

Iñiguez, Javier (2005), "La enseñanza de la genética, una propuesta didáctica para la educación secundaria obligatoria desde una perspectiva constructivista", Tesis de doctorado, Universidad de Barcelona, España.

Kozma, Robert y Joel Russell (2005), "Students becoming chemists: developing representational competence", en J. Gilbert (ed.), Visualization in Science Education, Switzerland, Springer, pp 121-146.

Lee, Hee-Sun, Lydia Liu Ou y Marcia Linn (2011), "Validating measurement of knowledge integration in science using multiple-choice and explanation items", Applied Measurement in Education, vol. 24, núm. 2, pp. 115-136.

Lewis, Jenny, John Leach y Colin Wood-Robinson (2000), "All in the genes? Young people's understanding of the nature of genes", fournal of Biological Education, vol. 34, núm. 2, pp. 74-79.

Magana, Alejandra J. y Sadhana Balachandran (2017), "Students' development of representational competence through the sense of touch", Fournal of Science Education and Technology, vol. 26, núm. 3, pp. 332-346.

Martí, Eduardo y Mercé García-Mila (2007), "Cambio conceptual y cambio representacional desde una perspectiva evolutiva: la importancia de los sistemas externos de representación", en J. I. Pozo y F. Flores (eds.), Cambio conceptual y representacional en el aprendizaje y la enseñanza de la ciencia, Madrid, Antonio Machado Libros, pp. 91-106.

Nersessian, Nancy (1990), Faraday to Einstein: Constructing meaning in scientific theories, Dordrecht, Kluwer Academic Publishers.

Pende, Prajakt y Sanjay Chandrasekharan (2017), "Representational competence: toward a distributed and embodied cognition account", Studies in Science Education, vol. 57, núm. 1, pp. 1-43.
Prain, Vaughan y Russell Tytler (2013), "Representing and learning in science", en R. Tytler, V. Prain, P. Hubber y B. Waldrip (eds.), Constructing representations to learn in science, Rotterdam, Sense Publishers, pp. 1-14.

Rau, Martina A., Vincent Aleven y Nikol Rummel (2015), "Successful learning with multiple graphical representations and self-explanation prompts", fournal of Educational Psychology, vol. 107, núm. 1, pp. 30-46.

Rogers, Laurence y John Twidle (2013), "A pedagogical framework for developing innovative science teachers with ICT", Research in Science Eै Technological Education, vol. 31, núm. 3, pp. 227-251.

Sánchez-Lazo, Sheila, Leticia Gallegos-Cázares y Fernando Flores-Camacho (2015), "El aprendizaje de la química en los nuevos 'Laboratorios de ciencia para el bachillerato UNAM", Revista Iberoamericana de Educación Superior (RIES), vol. 6 núm. 17, pp. 38-57.

Savinainen, Antti, Asko Mäkynen, Pasi Nieminen y Jouni Viiri (2017), "The effect of using a visual representation tool in a teaching-learning sequence for teaching Newton's third law", Research in Science Education, vol. 47, núm. 1, pp. 119-135, <DOI: https://doi.org/10.1007/ s1 1165-015-9492-8>

Schnotz, Wolfgang y Maria Bannert (2003), "Construction and interference in learning from multiple representations", Learning and Instruction, vol. 13, pp. 141-156, <DOI: 10.1016/S0959-4752(02)00017-8>.

Soderberg, Patti y Frank Price (2003), "An examination of problem-based teaching and learning in population genetics and evolution using EVOLVE, a computer simulation", International Fournal of Science Education, vol. 25, núm. 1, pp. 35-55.

Tortosa, Montserrat (2013), "Aprendizaje sobre disoluciones reguladoras de $\mathrm{pH}$ mediante indagación guiada utilizando sensores", Enseñanza de las Ciencias, vol. 13, núm. 1, pp. 189-211.

Waldrip, Bruce y Vaughan Prain (2013), “Teachers' initial response to a representational focus", en R. Tytler, V. Prain, P. Hubber y B. Waldrip (eds.), Constructing 
representations to learn in science, Rotterdam, Sense Publishers, pp. 15-30.

Wilson, Mark (2005), Constructing measures: An item response modeling approach, Mahwah, NJ, Lawrence Erlbaum Associates.
Winter, James de, Mark Winterbottom y Elaine Wilson (2010), "Developing a user guide to integrating new technologies in science teaching and learning: Teachers' and pupils' perceptions of their affordances", Technology, Pedagogy and Education, vol. 19, núm. 2, pp. 261-267.

\section{Muestra de los cuestionarios: A) Fuerza y movimiento, B) Genética.}

A
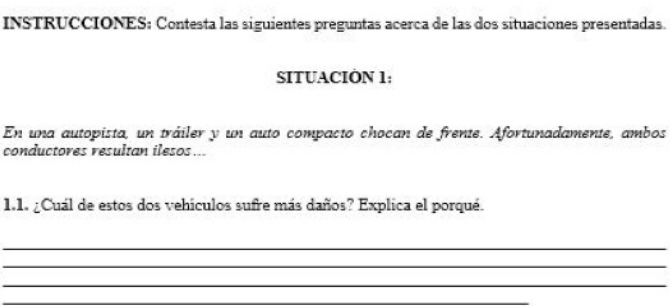
1.2. Cual da los dos conductores habrá sentido más el choque: el del triiler o el del auto
compacto? Explica el porqué.

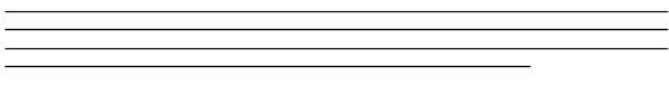
1.3. iEl impacto del trialer sobre el auto compacto es mayor, menor o igual que el impacto del
auto sobre el trailer? Explica el porque.

1.4. En la siguiente figura correspondiente a esta situación, dibuja la fuerza o las fuerzas presentes 1.4. En la siguiente figura correspondiente a esta situación, dibujaj la fuerza o las fuerzas presentes
en el momento del choque entre ambos vehiculos. Tambien, explica qué representa lo que has
B

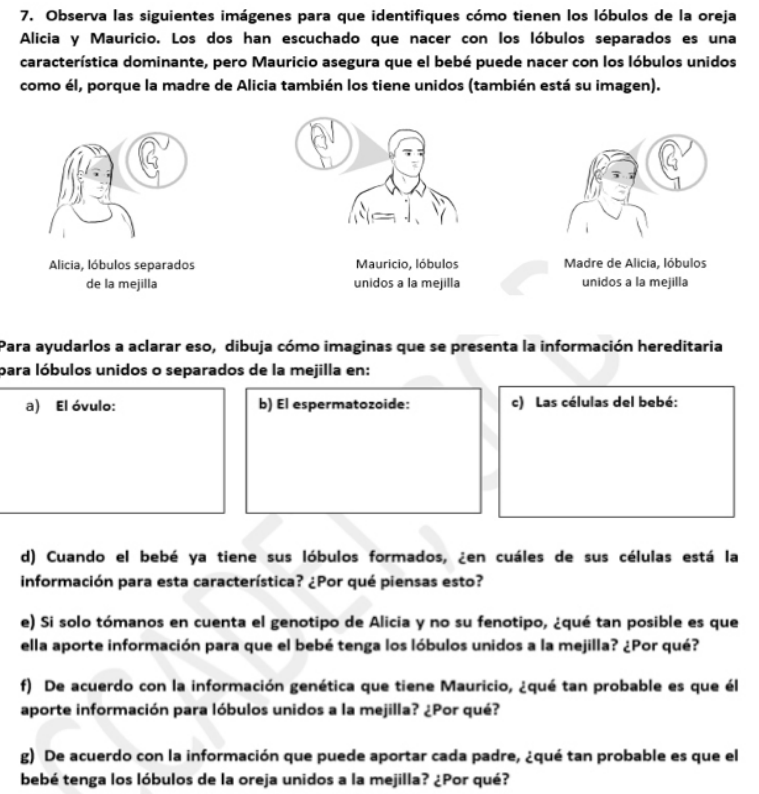

bebé tenga los lóbulos de la oreja unidos a la mejilla? ¿ Por qué?

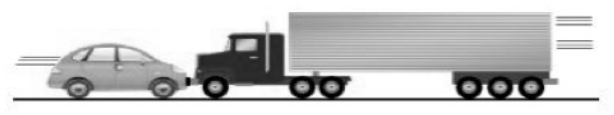

\section{Cómo citar este artículo:}

Flores-Camacho Fernando, Leticia Gallegos-Cázares, Beatriz-Eugenia García-Rivera y Araceli Báez-Islas (2019), “Efectos de los laboratorios de ciencias con TIC en la comprensión y representación de los conocimientos científicos en estudiantes del bachillerato en un contexto escolar cotidiano", en Revista Iberoamericana de Educación Superior (RIES), México, UNAM-IISUE/Universia, vol. x, Núm. 29, pp. 124-142, Dol: https://doi.org/10.22201/iisue.20072872e.2019.29.527 [consulta: fecha de última consulta]. 